REVIEW

\title{
The nasopharyngeal airway: dispelling myths and establishing the facts
}

\author{
K Roberts, H Whalley, A Bleetman
}

Emerg Med J 2005;22:394-396. doi: 10.1136/emj.2004.021402

The nasopharyngeal airway (NPA) is a simple airway adjunct used by various healthcare professionals. It has some advantages over the oropharyngeal airway (OPA) but despite this it appears to be used less frequently. This may be due to fears over intracranial placement in cases of possible basal skull fracture. This fear, promulgated by training, is based solely on two single case reports and relative risk needs to be put into clinical context. Widely taught methods of sizing NPAs are based upon the width of the patient's nostril or little finger, MRI data demonstrate that these methods are inaccurate. Ideal NPA length measured at nasal endoscopy correlates with subject height, this is independent of subject sex, and is a far more accurate determinant and easy to use in the clinical setting. Average height females require a Portex size 6 NPA and average height males a size 7 Portex NPA. This knowledge provides a rapid method of NPA selection.

See end of article for authors' affiliations

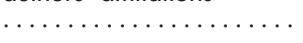

Correspondence to: Keith Roberts; dr_keith@hotmail.com

Accepted for publication 13 March 2005
$\mathrm{T}$ he nasopharyngeal airway (NPA) is a simple airway adjunct used in a number of healthcare disciplines, by staff trained to varying levels of competence in airway management. It has advantages over the oropharyngeal airway (OPA) as it can be used in patients with an intact gag reflex, trismus or oral trauma. Despite this, it is used less frequently than the OPA. ${ }^{12}$ This may be due to fears over intracranial placement in cases of possible basal skull fracture, promulgated in training. The basis for these concerns is revisited. Widely taught methods of sizing NPAs are based upon the width of the patient's nostril or little finger. ${ }^{34}$ The accuracy of these methods are reviewed in light of recent evidence. We sought a more accurate and rapid method of sizing NPAs.

\section{METHODS}

A Medline search was conducted with 'nasopharyngeal airway' as the key words.

\section{RESULTS}

The results identified 494 abstracts. Papers in English that reviewed indications and/or contraindications to NPA use and those that discussed methods of sizing NPAs were selected for review. The level of evidence for each paper referred to is presented where appropriate. (Level I, Strong evidence from at least one systematic review of multiple well-designed randomised controlled trials. Level II, Strong evidence from at least one properly designed randomised controlled trial of appropriate size. Level III, Evidence from well-designed trials such as non-randomised trials, cohort studies, time series, or matched case-controlled studies. Level IV, Evidence from well-designed non-experimental studies from more than one centre or research group. Level $\mathrm{V}$, Opinions of respected authorities, based on clinical evidence, descriptive studies or reports of expert committees.)

\section{DISCUSSION}

Sizing an NPA

Widely taught ${ }^{34}$ methods of sizing an NPA include comparing an NPA to the width of the patients little finger or nares. In paediatric practice it is also taught that an appropriate NPA will blanch the nares. ${ }^{5}$

In 2002 a study ${ }^{6}$ (Level III) assessed the validity of assessing subjects' little fingers or nares in order to size an appropriate NPA. Ten caucasian subjects attending for elective MRI of their skull had an additional sequence of scans performed at right angles to the nasal floor. From these the largest diameter NPA that would fit the subjects was calculated and compared with the subjects' little fingers and nares (as measured on the scans). The only measurement that closely correlated with the internal nasal anatomy was the medial-lateral distance of the subject's distal interphalangeal joint. The r-value of association on the right side was 0.02 and on the left 0.11 (values of 1 or -1 indicate complete correlation and 0 no correlation). The other measurements overestimated the appropriate airway in every instance. Therefore the currently taught methods of sizing NPAs are misleading.

In 1993, Stoneham ${ }^{7}$ (Level III) described that a correctly placed NPA will lie just above the epiglottis having separated the soft palate from the posterior wall of the oropharynx. This knowledge is vital if the NPA is to be sized correctly in patients: if the airway is too short it will fail to separate the soft palate from the pharynx and if too long it can either pass into the larynx and aggravate cough and gag reflexes or pass anterior to the epiglottis into the vallecula, a blind ending pouch, where paradoxical airway obstruction can occur if the NPA lumen is pressed against the soft tissues. Stoneham observed that the ideal NPA should lie approximately $10 \mathrm{~mm}$ above the epiglottis.

Abbreviations: NPA, nasopharyngeal; OPA,
oropharyngeal 
Thus the important factor in sizing a NPA is not the width of the tube, but rather the length. In the study Stoneham clearly identified a relationship between nares-epiglottis (N-E) distance and subject height. 120 adult caucasian subjects, under general anaesthesia, had their N-E distance measured. Subject height correlated with this distance and was independent of patient sex. Stoneham's average height females ( $\left.163 \mathrm{~cm} / 5^{\prime} 4^{\prime \prime}\right)$ had an N-E distance of $140 \mathrm{~mm} \mathrm{(95 \%} \mathrm{CI}$ $136.1-143.5 \mathrm{~mm})$ and males $\left(178 \mathrm{~cm} / 5^{\prime} 10^{\prime \prime}\right)$ an N-E distance of $159 \mathrm{~mm}$ (95\% CI 156.1-161.2 mm). Accepting that the airway should lie $10 \mathrm{~mm}$ above the epiglottis average height females in this study require a size 6 Portex $^{\mathrm{TM}}$ ( $130 \mathrm{~mm}$ long) NPA and similarly average height males a size 7 Portex $^{\text {TM }}$ (150 mm long) NPA. Because of the association between subject height and N-E distance a suitable NPA can be rapidly selected - for example, a tall male requires a size 8 NPA and a short male a size 6 . A tall female a size 7 and a short female a size 6 with the safety pin placed $1 \mathrm{~cm}$ from the flange.

A sizing chart based on Stoneham's work is presented in table 1 . The suggestions are based upon the evidence presented but ultimate selection will depend upon clinical response.

The association between subject height and N-E distance has also been identified in 73 Chinese subjects $^{8}$ (level III). In this series the male N-E distance was $160+/-10 \mathrm{~mm}$ and females $150+/-9 \mathrm{~mm}$. It appears that this trend also applies to paediatric practice. A study of 413 infants under 12 months also identified an association between subject height and nares-vocal cord distance with an r-value of 0.83 $(\mathrm{p}=0.0001)^{9}$ (Level III). This study also identified a very close association between nares-vocal cord distance and nose tip-earlobe distance ( see table 2). In order to place the NPA in infants the insertion length must therefore be slightly less than this anthropometric measurement.

\section{Contraindications and indications for use}

From reviews of paramedic practice, it is apparent that the use of the NPA outside of hospital is limited. In 2000, a review of national paramedic practice identified that only $27 \%$ of UK NHS Ambulance Trusts provide their paramedics with NPA's ${ }^{10}$ (Level IV). A further review in 2002, following the publication of national guidelines supporting the use of the NPA, found that this practice had increased, but only to 55\%. Similarly in 2003 only $21 \%$ of Ambulance Trusts would permit their paramedics to use NPA's in paediatric care ${ }^{11}$ (level IV).

The scale of NPA use in hospitals is less clear. However, it is the authors' observation that NPAs are used less frequently than OPAs. It is not clear why this might be the case. They are cheap and easy to use. It is possible that the fear of complications associated with the NPA has limited its use. It is widely taught that a suspected or known basal skull fracture is a contraindication to NPA placement. This is based upon two single case reports ${ }^{12}{ }^{13}$ (Level V). This contraindication needs to be interpreted in the appropriate setting: faced with airway obstruction and the possibility of a basal skull fracture a rescuer must secure the airway prior to any further management. In hospitals when a patient is known to have a basal skull fracture it is highly likely that this will have been diagnosed following a CT scan. In this scenario the issue of airway care will already have been addressed. Prior to a CT

\begin{tabular}{|c|c|}
\hline Subject Height & Suitable Portex ${ }^{\mathrm{TM}}$ NPA \\
\hline Short female & 6, pin $1 \mathrm{~cm}$ from flange \\
\hline Average* female/short male & 6 \\
\hline Tall female/average* male & 7 \\
\hline Tall male & 8 \\
\hline
\end{tabular}

Table 2 Infant weight and suggested insertion length of an appropriate NPA

\begin{tabular}{llll}
\hline $\begin{array}{l}\text { Body Weight } \\
(\mathrm{Kg})\end{array}$ & $\begin{array}{l}\text { Nosetip-Earlobe } \\
\text { distance }(\mathbf{c m})\end{array}$ & $\begin{array}{l}\text { Nares-Vocal cord } \\
\text { distance }(\mathbf{c m})\end{array}$ & $\begin{array}{l}\text { Approximate } \\
\text { insertion } \\
\text { length }(\mathbf{c m})\end{array}$ \\
\hline$<3$ & $7.6+/-1.3$ & $7.6+/-0.8$ & 7.0 \\
$3-6$ & $8.8+/-1.0$ & $8.8+/-0.7$ & 8.0 \\
$6-9$ & $10.0+/-0.8$ & $9.6+/-0.6$ & 9.0 \\
$9-12$ & $10.1+/-0.9$ & $10.2+/-0.5$ & 9.5 \\
\hline & & & \\
\hline
\end{tabular}

scan the diagnosis of a basal skull fracture can only be presumed by the presence or absence of clinical features. These include blood or cerebrospinal fluid from the ears or nose and bruising around the mastoid process or eyes. It must be appreciated that blood from the nose of patients with traumatic injuries is very common as is blood in the external ear (which has dripped into the ear rather than from within). CSF is a clear fluid and its identification is difficult, even in hospital. The simplest tests, the halo sign or the presence of glucose, are easy to perform but have a low sensitivity and specificity $^{14}{ }^{15}$ (Level III). The gold standard for laboratory diagnosis of CSF is the presence of beta-2-transferrin, ${ }^{14}$ this however, has no practical value as results are reported in 4 days. Bruising around the mastoid or eyes is also common following trauma and is more likely to be associated with soft tissue injury than a basal skull fracture.

Outside of hospital and particularly in low light or wet prehospital conditions, the recognition of CSF from the nose or ears is near impossible. Furthermore the development of this bruising will take some time and is unlikely to have developed when the patient is in the early stages of resuscitation whether this is in the pre-hospital environment or upon arrival to the Emergency Department. Therefore, the clinical features are vague and reliance upon their presence or absence may unnecessarily deter the use of the NPA.

The evidence for avoiding NPAs in cases of basal skull fracture is based solely upon two case reports. Securing the airway in an emergency takes precedence over a suspected basal skull fracture. The OPA is an obvious alternative but in practice a patient must have a conscious level much below that at which an NPA will be tolerated. If an OPA is placed and aggravates the gag reflex the risk of vomiting, aspiration, and increased intra-cranial pressure increases.

In teaching the use of the NPA, focus needs to shift from fear of contraindications to methods of safe placement to avoid intracranial placement. This needs to emphasise lifting the nares to reveal the nasal airway and the placement of the NPA parallel to the nasal floor, rather than upwards towards to the cribriform plate of the ethmoid bone. Lubrication, gentle rotation of the NPA and trying both nostrils are further methods that will ease insertion. These will reduce any risk of the often quoted but very infrequent complication of intracranial tube placement.

Other complications of NPA placement relate to soft tissue damage of the nasal mucosa. The mucosa is lined with pseudostratified columnar epithelium and vascular tissue that is similar to the erectile tissue of the penis. This tissue allows the normal cyclical variation in airway volume and alternates airflow between nostrils. It can be stripped off the nasal wall periosteum, causing bleeding. This is a relatively common problem associated with NPA insertion. If resistance is encountered when placing an NPA it is therefore recommended that the other nostril should be used to minimise this risk. A comparison of nasal soft tissue trauma as a result of NPA placement found no difference between the rates of haemorrhage between nurses or experienced anaethetists ${ }^{16}$ (Level III). 
Another rare complication is migration into the nasopharynx of the entire NPA ${ }^{17}{ }^{18}$ (Level V), it is for this reason that Portex NPA's have a flange and are supplied with a safety pin.

\section{CONCLUSIONS}

- The NPA is a simple piece of equipment that is easy to use and cheap. It is effective and has advantages over the OPA but appears to be used less frequently.

- Traditional methods of sizing NPAs place emphasis upon the width of the patient's nares or little finger, these are inaccurate and must be abandoned; tube length is more important. Evidence clearly demonstrates a relationship between NPA length to subject height, which is independent of sex. Average height females require a size 6 Portex ${ }^{\mathrm{TM}}$ NPA and males a size 7. Optimal and rapid sizing of the NPA can be modified from these average sizes to take account of the subject's height.

- The widely taught contraindication to NPA placement of a suspected basal skull fracture may be the reason that NPA use is less than that of the OPA. This extremely rare complication must be considered in relation to the potential benefit of simple and effective airway management. Fear of this complication will provide high false positive prediction of the fracture and may dissuade prehospital carers from using a very effective airway adjunct. Selecting an OPA in preference to an NPA in patients with an intact gag reflex risks vomiting and aspiration pneumonia. This maybe as equally grave as intracranial placement of a NPA and is certainly a more commonly encountered problem. Therefore methods of teaching must move from fear of complications to methods of safe placement of simple airway adjuncts and appropriate patient selection.

\footnotetext{
Authors' affiliations

K Roberts, Specialist Registrar in General Surgery, Walsall Manor Hospital H Whalley, Senior house officer in Intensive Care Medicine, Birmingham Heartlands Hospital, UK
}

A Bleetman, Consultant in Accident and Emergency Medicine, Birmingham Heartlands Hospital, UK

Competing interests: none declared

$K R$ and $A B$ are both HEMS doctors, Warwickshire and Northamptonshire Air Ambulance

\section{REFERENCES}

1 Roberts K, Allison KP, Porter KM. A review of emergency equipment carried and procedures performed by UK front line paramedics. Resuscitation 2003;58(2): 153-8.

2 Allison K, Porter K. Nasopharyngeal airways: an under-utilised pre-hospital resource. Pre-Hospital Immediate Care 2000;4(4):192-3.

3 Gwinnut C. Lecture notes on clinical anaesthesia, Blackwell Scientific Publications, Oxford, 1997:p39.

4 Greaves I, Porter KM, Ryan J. Trauma care manual. Arnold 2001:p41.

5 Advanced Paediatric Life Support Course Manual, BMJ books third ed. 2001:p36.

6 Roberts K, Porter K. How do you size a nasopharyngeal airway? Resuscitation 2003;56(1):19-23.

7 Stoneham MD. The nasopharyngeal airway. Assessment of position by fibreoptic laryngoscopy. Anaesthesia 1993;48(7):575-80.

8 Hwang CL, Luv KC, Wu TJ, et al. Estimation of the length of nasopharyngeal airway in Chinese adults. Ma Zui Xue Za Zhi 1990;28(1):49-54.

9 Shen CM, Soong WJ, Jeng MJ, et al. Nasopharyngeal tract length measurement in infants. Acta Paediatr Taiwan 2002:43(2):82-5.

10 Porter K, Allison K, Greaves I. Variations in equipment on UK front line. ambulances. Pre-hospital Immediate Care 2000;4:126-131.

11 Roberts K, Jewkes F, Whalley $\mathrm{H}$, et al. Equipment carried and procedures performed by UK paramedics upon paediatric patients. Resuscitation Submitted June, 2004

12 Muzzi DA, Losasso TJ, Cucchiara RF. Complication from a nasopharyngeal airway in a patient with a basilar skull fracture. Anaesthesiology 1991:74:366-8.

13 Schade K, Borzotta A, Michaels A. Intracranial malposition of nasopharyngeal airway. J Trauma 2000;49(5):967-8.

14 Constantino PD, Janecka IP. Cranial-base surgery. In: Head and Neck Surgery-Otolaryngology, second edn. In: Byron J, ed. Bailey. LipincottRaven, Philadelphia, 1998:1848-1853.

15 Chan DT, Poon WS, IP CP, et al. How useful is glucose detection in diagnosing cerebrospinal fluid leak? The rational use of CT and Beta-2 transferrin assay in detection of cerebrospinal fluid fistula? Asian J Surg 2004;27(1):39-42.

16 Chung $\mathrm{CH}$, Sum CW, Li HL, et al. Comparison of nasal trauma associated with nasopharyngeal airway applied by nurses and experienced anesthesiologists. Changgeng Yi Xue Za Zhi 1999;22(4):593-7.

17 Smith BL. Retained nasopharyngeal airway. Anaesthesia 1989;44(5):447.

18 Milam MG, Miller KS. Aspiration of an artificial nasopharyngeal airway. Chest 1988;93(1):223-4. 